Ferriz Valero, A.; García Martínez, S. y Arroyo Botella, J.M. (2019) Cooperative Methodologies decrease Disruptive Attitudes in Physical Education. Revista Internacional de Medicina y Ciencias de la Actividad Física y el Deporte vol. 19 (76) pp. 599-615 Http://cdeporte.rediris.es/revista/revista76/artretos1080.htm

DOI: $10.15366 /$ rimcafd2019.76.002

\title{
ORIGINAL
}

\section{METODOLOGÍA COOPERATIVA DISMINUYE LAS ACTITUDES DISRUPTIVAS EN EDUCACIÓN FÍSICA}

\section{COOPERATIVE METHODOLOGIES DECREASE DISRUPTIVE ATTITUDES IN PHYSICAL EDUCATION}

\author{
Ferriz Valero, A. ${ }^{1}$; García Martínez, S. ${ }^{1}$ y Arroyo Botella, J.M. ${ }^{2}$ \\ 1 Profesor Asociado Doctor, Dpto. Didáctica General y Didácticas Específicas en Universidad de \\ Alicante (España) alberto.ferriz@ua.es \\ 2 Profesor Asociado, Dpto. Didáctica General y Didácticas Específicas en Universidad de Alicante \\ (España) salvador.garcia@ua.es \\ 3 Independiente (España) imiguelarroyo.1991@gmail.com
}

\section{AGRADECIMIENTOS O FINANCIACIÓN}

Los autores afirman no existir ningún conflicto de interés ni financiación. Los autores agradecen la participación voluntaria del alumnado en el desarrollo del presente trabajo, así como a los centros educativos. Además, Miguel García-Jaén participó en este estudio subvencionado por un contrato predoctoral de la Generalitat Valenciana (ACIF/2016/048).

Código UNESCO / UNESCO Code: 5800 Pedagogía / Pedagogy Clasificación del Consejo de Europa / Council of Europe classification: 5. Didáctica y metodología / Didactics and methodology.

Recibido 26 de diciembre de 2017 Received December 26, 2017 Aceptado 3 de julio de 2018 Accepted July 3, 2018

\section{RESUMEN}

Las actitudes disruptivas suponen un gran problema para el profesorado en el transcurso de sus clases. Por ello, la finalidad del estudio es conocer y analizar las posibilidades que ofrece la metodología cooperativa como modelo pedagógico en la mejora de dichas actitudes. Se ha utilizado el cuestionario en su versión española validada de la versión corta original del Physical Education Classroom Instrument para la evaluación de conductas disruptivas en alumnado de secundaria. Se ha tomado una muestra de 114 sujetos de primer y segundo ciclo de ESO. Se realizaron dos sesiones de retos cooperativos en EF con un grupo (COOP); y con el otro grupo (CONTROL) se siguió la clase con normalidad. Tras la intervención, a diferencia del grupo control, se observan mejoras estadísticamente significativas en diversas variables tales como 
agresividad, desobediencia, irresponsabilidad y perturbación del ambiente de clase, favoreciendo así las relaciones interpersonales del grupo, tan sólo del grupo COOP.

PALABRAS CLAVE: Conductas disruptivas, nuevas metodologías, convivencia escolar, cooperación, trabajo en equipo, buenas actitudes, bullying.

\section{ABSTRACT}

Disruptive attitudes are frequently an important issue to be manage for teachers during their classes. In the study, we will try to analyse the possibilities offered by cooperative learning as a pedagogical model for the decrease in these disruptive attitudes. We used the validated Spanish version of the questionnaire of the original short version of the Physical Education Classroom Instrument for the evaluation of disruptive behaviour in high school students.

It was taken a sample of 114 subjects studying $1^{\text {st }}, 2^{\text {nd }}, 3^{\text {rd }}$ or 4 th grade. The study consisted on performing two sessions of cooperative challenges in a PE class with an experimental group (COOP); and with the other group (CONTROL) the classes were performed as usually. After the performance of these sessions, we found statistically significant improvement of the COOP group regarding several variables such as aggression, disobedience, irresponsibility and disruption of the classroom environment, encouraging the interpersonal relations of the COOP group, unlike the CONTROL group in which no improvement were found.

KEYWORDS: Disruptive behaviour, new methodologies, school, cohabitation, cooperation, teamwork, good attitudes, bullying.

\section{INTRODUCCIÓN}

Un aspecto que preocupa a los centros educativos es la calidad de su convivencia (Latorre \& Romero, 2014). Esta calidad de convivencia está presente de forma continua en las conversaciones, en las discusiones, en la prensa especializada; y resulta ser un problema de suma importancia que padece, en la actualidad, el sistema educativo (Uruñuela Nájera, 2007).

Los conflictos son inherentes a la naturaleza de las relaciones humanas y pueden originarse en cualquier proceso de convivencia, especialmente en la comunidad educativa. Por este motivo, se ha de aprender a abordarlos y debe ser un compromiso desde el ámbito educativo con objeto de minimizar los aspectos negativos de estos y favorecer el desarrollo socio-cognitivo de los estudiantes (Buchs, Butera, Mugny, \& Darnon, 2004; Frydenberg, 2004; Pérez Fuentes et al., 2011). Es importante afrontarlos positivamente en el momento que surjan como si fueran un reto a conseguir por el alumnado (Frydenberg, 2004; Pérez de Guzmán, Amador, \& Vargas, 2011).

Se podría pensar que los hechos que más afectan a esta convivencia son aquellos conocidos por su dureza y crueldad. Sin embargo, conductas como 
hablar en clase, apatía ante el profesorado o interrupciones constantes forman parte del principal problema con el que se encuentra el docente. Estas actitudes del alumnado son las llamadas conductas disruptivas, definiéndose como: todos aquellos conflictos o faltas de disciplina que atentan hacia el correcto desarrollo de la convivencia en el contexto educativo (Uruñuela Nájera, 2007). Estas conductas entorpecen el entorno de estudio y la calidad de la clase, así como produce inestabilidad psicológica por parte del profesorado, mermando su motivación e ilusión hacia su puesto de trabajo.

La literatura actual señala que estas conductas disruptivas se manifiestan de naturaleza leve o moderada, pero que se producen con mucha frecuencia. Además, parece no haber acuerdo en el reconocimiento de estas conductas entre el docente y el discente. De hecho, cada profesor las identifica de forma diferente, lo cual supone un problema a la hora de clasificarlas y, por ende, dotar de soluciones al colectivo escolar (Esteban, Fernández Bustos, Díaz Suárez, \& Contreras Jordán, 2012). En línea con esto, cada docente tiene visiones heterogéneas sobre los comportamientos considerados no apropiados y una capacidad distinta de tolerar los mismos (Siedentop, 1998).

Algunos estudios (Cothran \& Ennis, 1997; Cothran \& Kulinna, 2007; Supaporn, Dodds, \& Griffin, 2003) observan este nivel de discrepancia entre el alumnado y el profesorado. Estos autores informan de diferencias significativas en la identificación de las conductas y estrategias que, tanto el docente como el discente utilizan para el control de las mismas. Parece que, el profesorado presta atención a ciertos comportamientos que perturban el ambiente de clase, los cuales no son reconocidos por el alumnado en la mayoría de ocasiones. En otros casos, las conductas disruptivas suceden cuando los docentes no están presentes, encubriendo ese comportamiento no deseable del adulto. El género masculino suele ser más activo en el uso de estas conductas (Hastie \& Siedentop, 1999).

Pieron (1999) establece cuatro tipos de comportamientos conflictivos: en relación a la tarea, al profesor, a los compañeros y a los eventos. Otras clasificaciones atienden a criterios de gravedad (Kulinna, Cothran, \& Recualos, 2003) o al grado de perturbación que provocan en clase (Goyette, Doré, \& Dion, 2000). Algunos estudios centraron su atención en la percepción del profesorado (Kulinna, Cothran, \& Recualos, 2006) y establecen qué conductas entiende el profesor como conflictivas agrupándolas según su gravedad y tipo de conducta. Todas las conductas comentadas anteriormente son muy variadas entre sí. Sin embargo, presentan en común que dificultan la convivencia y se oponen a la tarea educativa propia de los centros de aprendizaje que constituye el proceso de enseñanza-aprendizaje (Esteban et al., 2012), la doble intencionalidad de los centros educativos. Esta cohesión es capital para el correcto desarrollo adolescente (Uruñuela Nájera, 2007).

En línea con lo anterior, Uruñuela (2007) divide estas conductas en dos dimensiones dentro del sistema educativo: la primera dimensión hace referencia al Centro de Aprendizaje, destacando aquellas conductas que atentan sobre el propio proceso de enseñanza dentro del aula; y la segunda al Centro de convivencia que hace referencia a aquellas conductas que minan la convivencia 
entre la comunidad que forma parte del contexto educativo. Por un lado, dentro del grupo de conductas referidas al Centro de Aprendizaje, el primer tipo de conducta disruptiva es la falta de rendimiento. Se refieren a las actitudes de pasividad, desinterés y apatía que suele manifestar el alumnado, como cuando no traen el material necesario para el trabajo en clase o no hacer tareas para casa, estar fuera de la clase y/o en sitios no autorizados durante el horario escolar, etc. El segundo tipo de conducta, se denomina molestar en clase, se puede presentar de muchas formas y es una de las que más preocupa al profesorado. Abarca conductas como: hablar y no guardar el silencio necesario para las explicaciones, moverse libremente por el aula o gimnasio, boicotear la autoridad del docente e impedir el normal desarrollo de la clase, etc. Por último, la tercera categoría de conducta se refiere al absentismo y abarca desde pequeños retrasos reiterados hasta la ausencia continuada. Por otro lado, en el grupo de conductas referidas al Centro de Convivencia se presentan tres: la falta de respeto, el conflicto de poder y la violencia. Este grupo de conductas presenta un abanico muy amplio, e incluso algunas conductas pueden ser clasificadas en los dos grupos al mismo tiempo. Por falta de respeto se pueden observar conductas como estallar de risa en la ejecución de una habilidad motriz de un-a compañero-a. Por conflicto de poder, son aquellas conductas de enfrentamiento del alumnado con el profesorado, quedando por encima y desafiando sus indicaciones, así como el incumplimiento de sanciones impuestas por el docente. La tercera categoría es la violencia, la cual puede expresarse hacia el material, hacia los iguales o hacia el docente, pudiendo ser verbal o física.

Una de las causas destacadas sobre las demás a la hora de determinar qué produce en los jóvenes la necesidad de actuar disruptivamente es el contexto familiar. La manera, el afecto y el tiempo dedicado al cuidado del tutelado resulta ser fundamental sobre el comportamiento que cada individuo lleva a cabo, no siendo la actitud disruptiva una excepción (Cetina, 2011; Navarrete, Lucía \& Ossa, 2013). Otras situaciones como separaciones o relaciones problemáticas entre cónyuges pueden incrementar los problemas conductuales de los adolescentes. Sin embargo, el profesor puede incurrir en ese comportamiento, influyendo en el modo que tiene el alumnado de relacionarse entre sí, de manera que un buen clima de enseñanza puede contribuir a la adquisición de competencias pro-sociales (Sánchez, 2012).

La Educación Física (en adelante, EF) hace posible, desde su especial capacidad de interacción, influir de una manera significativa en el comportamiento del alumnado. Se puede generar empatía y, por ende, un clima de respeto y ayuda mutua. Además, su carácter lúdico convierte el contexto físico-deportivo en el escenario idóneo para que tenga lugar el proceso de aprendizaje (Esteban et al., 2012; Monzonís \& Capllonch, 2014).

Las conductas disruptivas han sido controladas habitualmente mediante el uso de un modelo educativo autoritario. Sin embargo, en la actualidad, se opta por otro tipo de opciones más aceptadas por los expertos, como pueden ser modelos con mayor grado de libertad y modelos funcionales donde el alumnado aprenda la utilidad y la necesidad de actuar correctamente en lugar de ser obligado a hacerlo, sin exponer argumento alguno (Prat Grau \& Soler Prat, 2002). 
Las clases de EF no pasan indiferentes ante estos problemas, de hecho, ocurre tan habitualmente como en las asignaturas que se imparten en el aula (White \& Bailey, 1990). Del mismo modo que en el resto de materias, estas conductas entorpecen la organización y el funcionamiento de las sesiones de EF (Fernandez-Balboa, 1990) generando frustración entre profesorado y alumnado (Fejgin, Talmor, \& Erlich, 2005).

Algunos autores (Brown, 1990; Grineski, 1996; Omeñaca \& Ruiz, 1999), hacen referencia al juego cooperativo como una actividad que permite liberar al alumnado de aspectos negativos, principalmente relacionados con la competición, la exclusión, las reglas inflexibles y las agresiones. Parece que estos juegos cooperativos favorecen la inclusión, la libertad de decisión, el compañerismo, la aceptación de diferencias, la resolución de conflictos y, en definitiva, contribuye a la disipación de conductas disruptivas.

La actividad física cooperativa tiene mayor capacidad de reducir la agresividad $y$ las conductas disruptivas que las actividades competitivas. Bay-Hinitz, Peterson, \& Quilitch (1994) demostraron que, tras la realización de un programa de juegos cooperativos, el alumnado sufrió un aumento de las conductas de ayuda-cooperación y una disminución significativa de las conductas agresivas. Estos resultados fueron contrarios a aquéllos que provocaron un programa de juegos competitivos. A su vez, Finlinson (1997) durante un estudio similar, observó que las conductas prosociales se incrementaron tras la aplicación de un programa de juegos cooperativos.

Los juegos cooperativos se definen como aquellos juegos en que los jugadores interactúan entre sí, potenciando un clima de ayuda mutua, para contribuir a alcanzar objetivos comunes (Garaigordobil Landazabal, 2004). De este modo, la realización de juegos cooperativos supone un importante recurso para el educador a la hora de lograr una maduración emocional en el alumnado, siendo útil su educación tanto en el contexto de educación formal como en actividades de ocio y tiempo libre.

\section{OBJETIVOS}

Por estas y otras razones, este estudio se plantea con el fin de observar el efecto de una intervención a través de metodologías cooperativas en EF sobre algunas variables relacionadas con la aparición de conductas disruptivas. Como objetivo secundario, se pretende observar las diferencias atribuibles al género en la aparición de esas conductas disruptivas.

\section{MATERIAL Y MÉTODOS}

Se trata de un estudio de corte cuasi experimental doble ciego, cuyo muestreo fue por conglomerados no probabilístico. 


\section{MUESTRA}

El estudio tuvo lugar en dos centros públicos de Educación Secundaria Obligatoria de la provincia de Alicante (España) catalogados como centros de atención educativa singular. Inicialmente participaron 184 sujetos pertenecientes a aulas de $3^{\circ}$ y $4^{\circ}$ ESO, quedando excluidos del mismo a 80 sujetos por cumplir los criterios exclusión. Estos criterios de exclusión fueron: (1) No contestar los datos de codificación o algún ítem en particular, (2) no realizar alguno de los dos test y (3) el absentismo. Las características de la muestra quedan reflejadas en la siguiente Tabla 1.

Todos los participantes fueron informados de su participación en el estudio, así como el propio centro escolar, siendo estos quienes dieron su aprobación para publicar los datos del alumnado de forma anónima tras firmar un acuerdo de confidencialidad con las personas encargas de realizar el presente estudio. Una vez obtenida la autorización por parte del centro, la muestra fue dividida en dos grupos según recibieran intervención a través de metodología cooperativa (grupo Coop) 0 , por el contrario, siguieran el transcurso de sus clases como habitualmente (grupo Control).

Tabla 1. Características del muestreo (género de los participantes y excluidos)

\begin{tabular}{lcccccc}
\hline GRUPO & Curso & $\begin{array}{c}\text { Muestra } \\
\text { Inicial }\end{array}$ & Fem & Masc & Excluidos & Total \\
\hline CONTROL & $3^{\circ}$ ESO & 44 & 13 & 16 & 15 & $\mathbf{2 9}$ \\
COOP & $4^{\circ}$ ESO & 49 & 9 & 11 & 29 & $\mathbf{2 0}$ \\
& $3^{\circ}$ ESO & 29 & 19 & 12 & 8 & $\mathbf{3 1}$ \\
& $4^{\circ}$ ESO & 62 & 16 & 18 & 28 & $\mathbf{3 4}$ \\
& Total & $\mathbf{1 8 4}$ & $\mathbf{5 7}$ & $\mathbf{5 7}$ & $\mathbf{8 0}$ & $\mathbf{1 0 4}$ \\
\hline
\end{tabular}

\section{PROCEDIMIENTO}

La intervención se llevó a cabo a través de dos sesiones las cuales forman parte de una Unidad Didáctica de retos cooperativos en el grupo experimental. El grupo control realizó las clases de EF con normalidad, poniendo en práctica una Unidad Didáctica de Juegos populares a través de estilos de enseñanza tradicionales (mando directo modificado y asignación de tareas), realizando el cuestionario sobre las conductas disruptivas en el aula. Las actividades de enseñanza-aprendizaje que se llevaron a cabo durante las sesiones en el grupo de retos cooperativos fueron:

- Atravesar el aro horizontal: el propósito es que todo el alumnado ha de traspasar un aro de arriba a abajo sin tocarlo con ninguna parte del cuerpo.

- El balón imposible: se trata de trasladar una pelota gigante, sin tocar el suelo entre el punto de partida y el de llegada. Además, todos los miembros del grupo han de tocar la pelota con una parte del cuerpo distinta. 
- La pelota se va de marcha: se ha de mover una pelota por un determinado recorrido mediante golpeos. Cada miembro tan sólo tendrá un tiro.

- Giro de colchoneta: se trata de dar la vuelta a la colchoneta, con todos los miembros del grupo subidos encima y sin tocar el suelo.

- Paz: el grupo ha de construir con esterillas la palabra paz. Todo el grupo ha de estar pisando las esterillas y nadie podrá tocar el suelo en ningún momento.

- Sacar el jamón del bocadillo: se colocan tres colchonetas, una encima de la otra sin sobresalir. Todo el equipo se sube encima. El objetivo es sacar la colchoneta que se encuentra en medio, sin tocar el suelo.

- El ciempiés viajero: todo el grupo unido mediante el agarre que elijan, debe hacer un circuito propuesto.

Las sesiones fueron planteadas de manera que los participantes debían resolver con éxito 7 retos (4 y 3 por sesión), en grupos que oscilaron entre 5 y 7 componentes (Velázquez Callado, 2004). Finalmente, al concluir la sesión se realizaba una reflexión del profesor y alumnado conjuntamente, aportando opiniones personales relacionadas con el comportamiento, durante la consecución de los retos (Gil Espinosa, Chillón Garzón, \& Delgado Noguera, 2016). El esquema del procedimiento se puede observar a continuación.

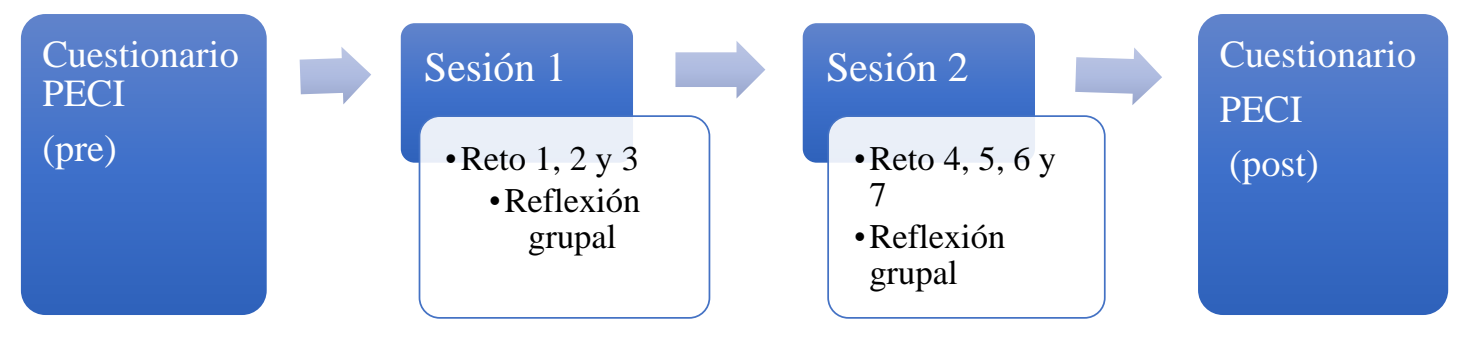

Ilustración 2. Esquema del procedimiento del estudio.

\section{MATERIAL}

Por un lado, se utilizó un cuestionario destinado a evaluar las conductas disruptivas del grupo. Este cuestionario es una versión española validada de la versión corta original del Physical Education Classroom Instrument para la medición de conductas disruptivas en alumnado de secundaria (GraneroGallegos \& Baena-Extremera, 2016). Este cuestionario consiste en la evaluación de 5 variables relacionadas con las conductas disruptivas: (1) agresividad, (2) irresponsabilidad y bajo compromiso, (3) desobediencia de las normas, (4) perturbador del ambiente de clase y (5) bajo autocontrol personal; a través de una escala likert del 1 al 5 . Los participantes debían contestar sinceramente acerca de sí mismos.

Por otro lado, se aplicó una Unidad Didáctica titulada "Retos cooperativos". En esta Unidad Didáctica se explicó al alumnado la definición de reto cooperativo, 
entendiéndose como un desafío motriz y cognitivo con diferentes soluciones, siendo un objetivo común del grupo (5-7 alumnos-as). Cuantos más retos supere el grupo, mejor calificación obtendrá el mismo, siempre respetando las normas y el fair-play; $y$ siguiendo el lema: para, piensa y actúa. El alumnado fue informado sobre los criterios de evaluación seguidos por el docente. Asimismo, dentro del grupo se definieron una serie de roles que se describen en la siguiente Tabla 2.

Tabla 2. Roles establecidos para el alumando en el grupo cooperativo

\begin{tabular}{|c|c|}
\hline ROL & DESCRIPCIÓN \\
\hline Secretario & Leer los retos y recordar las normas. \\
\hline Animador 1,2 y 3 & $\begin{array}{l}\text { Dinamizar la actividad y animar al grupo en todo momento a } \\
\text { superar los retos. }\end{array}$ \\
\hline Moderador & $\begin{array}{l}\text { Organizar los turnos de palabra cuando se proponen ideas para } \\
\text { resolver el reto. }\end{array}$ \\
\hline Tiempo & $\begin{array}{l}\text { Controlar el tiempo disponible para cada reto y avisar a los } \\
\text { compañeros-as. }\end{array}$ \\
\hline Orden & $\begin{array}{l}\text { Proporcionar y recoger el material. Organizar al grupo y } \\
\text { supervisar que todos participen en la consecución del reto. }\end{array}$ \\
\hline
\end{tabular}

Finalmente, se realizó un grupo de reflexión sobre la práctica realizada, donde cada alumno-a aportaba su punto de vista en función del rol desempeñado en la sesión. Se utilizó un registro anecdotario para la recogida de datos. En este grupo de discusión, se planteó la utilidad de las metodologías cooperativas como herramienta para reducir la incidencia de conductas no deseables durante la sesión. Este registro fue analizado posteriormente a través de un proceso de codificación siguiendo la metodología de análisis cualitativa.

\section{ANÁLISIS ESTADÍSTICO}

Los datos fueron sometidos a un análisis estadístico univariante para muestras no paramétricas, concretamente se utilizó la prueba de Wilcoxon y la U de MannWhitney. Se hizo uso del programa estadístico SPSS para MAC (IBM® SPSS® Statistics Versión 24.0.0.0).

\section{RESULTADOS}

En primer lugar, se presentan los resultados estadísticos obtenidos tras la aplicación de la prueba de Wilcoxon, en tres apartados diferenciados, para facilitar su comprensión e interpretación: (1) diferencias entre grupo, (2) diferencias entre grupo y nivel y (3) diferencias entre grupo y género.

\section{(1) CONTROL VS COOP}

En la Tabla 3 se observan las diferencias entre el pretest y el postest para los grupos del estudio (Control vs Coop). Se observan diferencias significativas 
$(p<0,001)$ para el grupo experimental en todas las variables dependientes excepto en Bajo autocontrol, es decir, el grupo que trabajó actividades y retos cooperativos mejoró su tendencia hacia las conductas disruptivas.

Tabla 3. Resultados obtenidos de la aplicación de la prueba Wilcoxon (pretest vs postest) en la versión española del cuestionario Physical Education Classroom Instrument (promedio \pm DT)

\begin{tabular}{lcccccc}
\hline & \multicolumn{3}{c}{ Control } & \multicolumn{3}{c}{ Coop } \\
& Pre & post & Z & Pre & post & Z \\
\hline Agresividad & $1,56 \pm 0,52$ & $1,48 \pm 0,49$ & $-1,173$ & $1,44 \pm 0,54$ & $1,19 \pm 0,54$ & $-3,742^{* *}$ \\
Irresponsabilidad & $1,97 \pm 0,76$ & $1,87 \pm 0,72$ & $-0,563$ & $1,83 \pm 0,63$ & $1,43 \pm 0,50$ & $-4,846^{* *}$ \\
Desobediencia & $1,52 \pm 0,54$ & $1,48 \pm 0,52$ & $-0,520$ & $1,57 \pm 0,74$ & $1,34 \pm 0,53$ & $-3,525^{* *}$ \\
Perturbación & $1,55 \pm 0,58$ & $1,65 \pm 1,27$ & $-0,223$ & $1,34 \pm 0,43$ & $1,20 \pm 0,35$ & $-3,369^{* *}$ \\
Bajo autocontrol & $1,43 \pm 0,57$ & $1,33 \pm 0,45$ & $-1,414$ & $1,24 \pm 0,40$ & $1,18 \pm 0,43$ & $-1,478$ \\
\hline & \multicolumn{5}{c}{$p<0,05$} & ${ }^{* *} p<0,01$
\end{tabular}

(2) GRUPO*NIVEL

Si se observan los mismos datos teniendo en cuenta el nivel ( $3^{\circ}$ ó $4^{\circ}$ ) , se puede observar que, de nuevo, el grupo experimental obtiene diferencias estadísticamente significativas en todas las variables y niveles, excepto una tendencia a la significatividad para las variables agresividad y Bajo autocontrol en 3ํㅡㄴ ESO $(p=0,58$ y 0,65 , respectivamente).

Tabla 4. Resultados obtenidos de la prueba Wilcoxon (grupo*nivel)

\begin{tabular}{|c|c|c|c|c|c|}
\hline & & & trol & & \\
\hline & & $\mathbf{Z}$ & $\begin{array}{c}\text { Sig. } \\
\text { asintótica } \\
\text { (bilateral) }\end{array}$ & $\mathbf{Z}$ & $\begin{array}{c}\text { Sig. } \\
\text { asintótica } \\
\text { (bilateral) }\end{array}$ \\
\hline Agresividad & 3 ESO & $-0,206^{b}$ & ,837 & $-1,894^{b}$ & ,058 \\
\hline & $4^{\circ} \mathrm{ESO}$ & $-0,949^{b}$ & ,343 & $-3,571^{b}$ & ,000 \\
\hline Irresponsabilidad & $3^{\circ}$ ESO & $-0,180^{b}$ & ,857 & $-3,216^{b}$ & ,001 \\
\hline & $4^{\circ} \mathrm{ESO}$ & $-0,637^{b}$ &, 524 & $-3,694^{b}$ & ,000 \\
\hline Desobediencia & $3^{\circ}$ ESO & $-1,101^{c}$ & ,271 & $-2,094^{b}$ & ,036 \\
\hline & $4^{\circ} \mathrm{ESO}$ & $-1,785^{b}$ & 074 & $-3,018^{b}$ & ,003 \\
\hline Perturbación & 3 ESO &,$- 0960^{c}$ & ,337 & $-2,165^{b}$ & ,030 \\
\hline & $4^{\circ} \mathrm{ESO}$ & $-1,701^{b}$ & ,089 & $-2,944^{b}$ & ,003 \\
\hline Bajo autocontrol & $3^{\circ}$ ESO & $-1,079^{b}$ & ,281 & $-0,458^{b}$ & ,647 \\
\hline & $4^{\circ}$ ESO & $-0,848^{b}$ & ,396 & $-2,308^{b}$ & ,021 \\
\hline
\end{tabular}

a. Prueba de rangos con signo de Wilcoxon.
b. Se basa en rangos positivos.
c. Se basa en rangos negativos.

(3) GRUPO^GÉNERO 
Tal y como se puede apreciar en la Tabla 5, son estadísticamente significativas todas las variables evaluadas independientemente del género, excepto para la Perturbación en el género masculino $(p=0,123)$ y el Bajo autocontrol en ambos géneros.

Tabla 5. Resultados obtenidos de la prueba Wilcoxon (grupo*género)

\begin{tabular}{|c|c|c|c|c|c|}
\hline & & \multicolumn{2}{|c|}{ Control } & \multicolumn{2}{|c|}{ Coop } \\
\hline & & $\mathbf{Z}$ & $\begin{array}{c}\text { Sig. } \\
\text { asintótica } \\
\text { (bilateral) }\end{array}$ & $\mathbf{Z}$ & $\begin{array}{c}\text { Sig. } \\
\text { asintótica } \\
\text { (bilateral) }\end{array}$ \\
\hline \multirow[t]{2}{*}{ Agresividad } & Masc & $-0,726^{b}$ & ,468 & $-2,032^{b}$ & ,042 \\
\hline & Fem & $-1,006^{b}$ & ,314 & $-3,402^{b}$ &, 001 \\
\hline \multirow[t]{2}{*}{ Irresponsabilidad } & Masc & $-0,489^{b}$ & ,625 & $-2,562^{b}$ & ,010 \\
\hline & Fem & $-0,246^{b}$ & ,806 & $-4,179^{b}$ &, 000 \\
\hline \multirow[t]{2}{*}{ Desobediencia } & Masc & $-0,506^{b}$ & ,613 & $-2,225^{b}$ & ,026 \\
\hline & Fem & $-0,262^{b}$ & ,794 & $-2,856^{b}$ &, 004 \\
\hline \multirow[t]{2}{*}{ Perturbación } & Masc & $-1,071^{b}$ & ,284 & $-1,543^{b}$ & ,123 \\
\hline & Fem & $-0,731^{c}$ & ,465 & $-2,992^{b}$ &, 003 \\
\hline \multirow[t]{2}{*}{ Bajo autocontrol } & Masc & $-1,798^{b}$ & ,072 & $-0,922^{b}$ & ,357 \\
\hline & Fem & $-0,362^{c}$ & ,717 & $-1,259^{b}$ & ,208 \\
\hline
\end{tabular}

a. Prueba de rangos con signo de Wilcoxon.

b. Se basa en rangos positivos.

c. Se basa en rangos negativos.

Finalmente, no hubo diferencias significativas iniciales (ni finales) en las variables evaluadas entre sexos, excepto en el Bajo autocontrol al inicio del estudio, que es peor en el género masculino (ver Tabla).

Tabla 6. Resultados obtenidos de la prueba U-Mann Whitney (variable de agrupación = género)

\begin{tabular}{lcccc}
\hline & \multicolumn{2}{c}{ Pre } & \multicolumn{2}{c}{$\begin{array}{c}\text { Post } \\
\text { Sig. asintótica } \\
\text { (bilateral) }\end{array}$} \\
\hline Agresividad & $-0,791$ &, 429 & $-1,299$ &, 194 \\
Irresponsabilidad & $-0,430$ &, 667 & $-0,647$ &, 517 \\
Desobediencia & $-0,324$ &, 746 & $-0,206$ &, 837 \\
Perturbación & $-1,321$ &, 186 & $-0,441$ &, 659 \\
Bajo autocontrol & $-3,039$ &, 002 & $-1,251$ &, 211 \\
\hline
\end{tabular}

a. Prueba de rangos con signo de Wilcoxon.

b. Se basa en rangos positivos. 
En segundo lugar, no se observaron diferencias estadísticamente significativas entre la puntuación obtenidas por cada uno de los siete retos conseguidos entre los distintos grupos, ni siquiera entre niveles ( $3^{\circ} \mathrm{ESO}$ vs $4^{\circ} \mathrm{ESO}$ ).

Finalmente, se destacan algunas reflexiones relevantes de naturaleza cualitativa extraídas de comentarios y afirmaciones realizadas por el alumnado en la reflexión grupal realizada al final de cada sesión, recogidas en un registro anecdotario.

Tabla 7. Resultados del análisis cualitativo del registro anecdotario

Percepción del alumnado sobre la sesión de retos cooperativos y su eficacia como metodología docente para reducir las conductas disruptivas

\begin{tabular}{|c|c|c|c|}
\hline & FA & $\% F A$ & Ejemplos \\
\hline $\begin{array}{l}\text { Más eficaz y } \\
\text { divertida }\end{array}$ & 7 & 58.33 & $\begin{array}{l}\text { Nos ha gustado porque no ha habido peleas } \\
\text { serias entre nosotros y se ha tenido en cuenta } \\
\text { la opinión de todos (Moderadora } 4,3^{\circ} E S O \text { ) }\end{array}$ \\
\hline $\begin{array}{l}\text { Más eficaz pero } \\
\text { menos divertida }\end{array}$ & 1 & 8.33 & $\begin{array}{l}\text { Yo me lo paso mejor cuando juego al futbol } \\
\text { porque depende sólo de mí. (Moderador } 9 \text {, } \\
4^{\circ} E S O \text { ) }\end{array}$ \\
\hline $\begin{array}{l}\text { Similar a otras } \\
\text { sesiones }\end{array}$ & 2 & 16.66 & $\begin{array}{l}\text { No hemos notado diferencias con otras clases } \\
\text { (Moderador } 11,4^{\circ} \text { ESO) }\end{array}$ \\
\hline $\begin{array}{l}\text { Menos eficaz } \\
\text { pero divertida }\end{array}$ & 1 & 8.33 & $\begin{array}{l}\text { Han sido juegos divertidos pero en mi grupo se } \\
\text { han peleado más por conseguir los puntos. } \\
\text { (Moderador } 1,3^{\circ} \text { ESO) }\end{array}$ \\
\hline $\begin{array}{l}\text { Menos eficaz y } \\
\text { menos divertida }\end{array}$ & 0 & 0 & 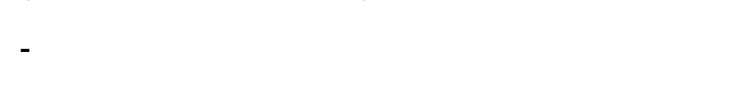 \\
\hline
\end{tabular}

\section{DISCUSIÓN}

En línea con algunos autores (Sánchez, 2012), los resultados del presente estudio subrayan la importancia de la figura del profesorado de EF en la mejora de actitudes negativas usuales en el alumnado, mediante la planificación de actividades específicas como son los retos cooperativos (Velázquez Callado, 2004). Estas propuestas o retos grupales son intrínsecamente capaces de mejorar las actitudes disruptivas del alumnado en 4 de 5 variables diferentes (agresividad, Irresponsabilidad, Desobediencia y Perturbación del ambiente de clase) de manera muy significativa estadísticamente, en consonancia con los resultados observados por Bay-Hinitz et al., (1994) y Finlinson (1997). Además, en el grupo de discusión se observa que la percepción mayoritaria del alumnado considera este tipo de sesiones más eficaces y más divertidas.

La materia de EF en Secundaria, pues, tiene un papel relevante en la regulación y modificación de actitudes disruptivas (Esteban et al., 2012; Monzonís \& Capllonch, 2014). Tal y como apuntan expertos en la materia (Cothran \& Ennis, 1997; Cothran \& Kulinna, 2007; Supaporn et al., 2003), el alumnado usualmente no es consciente de la problemática que supone este tipo de conductas en el aula. Este hecho, hace necesaria una correcta realización de programas enfocados a la reducción de comportamientos que puedan ser nocivos para la convivencia del centro como las actividades y juegos cooperativos con una reflexión final, donde el alumnado pueda concienciarse mediante esas 
reflexiones sobre el problema real que generan estos comportamientos repetitivos e incesantes (Uruñuela Nájera, 2007). En este sentido, en el grupo de discusión tuvieron oportunidad de reflexionar sobre los conflictos que habitualmente pueden surgir en el desarrollo de las sesiones de EF y como esta metodología cooperativa es eficaz y necesaria para reducir la incidencia de conductas disruptivas, así como la autoconcienciación de la responsabilidad individual para la convivencia colectiva.

El uso de actividades y metodologías cooperativas o la utilización de estrategias para conseguir un correcto clima de respeto en las aulas debe realizarse de manera diaria a lo largo del curso escolar (Velázquez Callado, 2004), incluyéndolo de manera transversal en las unidades didácticas no específicas.

Es evidente que los resultados obtenidos en este estudio son muy relevantes para la asignatura de EF en Secundaria y para el centro educativo en general, teniendo en cuenta que la intervención didáctica ha sido tan sólo de dos sesiones y dos niveles, aunque fuertemente conflictivos. Es posible que, una extensión más intensa y prolongada (Gallego, 2012; Velázquez Callado, 2004) de juegos o retos cooperativos puede, incluso, mejorar significativamente la variable que se ha mantenido sin cambios, el Bajo autocontrol. Sin embargo, para esa afirmación hacen falta más estudios de esta índole.

Según algunos autores (Hastie \& Siedentop, 1999), los hombres demuestran ser más conflictivos dentro del horario escolar. Sin embargo, la última parte de los resultados de nuestro estudio no apoyan esta afirmación excepto en la variable Bajo autocontrol $(p=0,002)$. Tan sólo en la variable Perturbación del ambiente, observamos que los chicos no mejoran significativamente tras la intervención, pero no se puede afirmar una diferencia atribuida al género.

Para futuras líneas de investigación, es necesario contrastar con similares intervenciones los datos obtenidos en el presente estudio y, además, realizar una intervención con estilos de enseñanza autoritarios que hagan uso de castigos, comparando la eficacia y eficiencia de los juegos cooperativos con los distintos tipos de estilo de enseñanza, así como la percepción del alumnado relacionada con estos estilos.

\section{CONCLUSIONES}

Las conductas disruptivas suponen uno de los problemas principales en la integridad del centro como lugar de convivencia y para el desarrollo óptimo de las clases. Por eso, estas conductas pueden verse reducidas teniendo en cuenta las siguientes premisas:

- La realización de un programa de retos cooperativos en EF de, al menos, dos días de intervención, puede adoptar cambios positivos (aparición de conductas prosociales, disminución de conflictos interpersonales, etc.) a corto plazo en el alumnado de primer y segundo ciclo de ESO. 
- La aplicación de esta estrategia educativa es eficaz tanto para el alumnado masculino como para el femenino y mejora el aprendizaje cooperativo entre el alumnado.

- El alumnado percibe mayoritariamente la utilidad de esta metodología para reducir la incidencia de conflictos, además de apreciar estas sesiones como divertidas y motivantes.

\section{REFERENCIAS BIBLIOGRAFICAS}

Bay-Hinitz, A. K., Peterson, R. F., \& Quilitch, R. H. (1994). Cooperative games: a way to modify aggressive and cooperative behaviors in young children. Journal of Applied Behavior Analysis, 27(3), 435-446. https://doi.org/10.1901/jaba.1994.27-435

Brown, G. (1990). Que tal si jugamos... otra vez. (2nd ed.). Caracas: Guarura Ediciones.

Buchs, C., Butera, F., Mugny, G., \& Darnon, C. (2004). Conflict Elaboration and Cognitive Outcomes. Theori into Practice, 43(1), 6-13. https://doi.org/10.1353/tip.2004.0002

Cetina, A. S. (2011). Problemas familiares generadores de conductas disruptivas en alumnos, 2, 423-432.

Cothran, D., \& Ennis, C. (1997). Students' and Teachers' Perceptions of Conflict and Power. Teaching and Teacher Education, 53(9), 541-553. http://doi.org/10.1017/CBO9781107415324.004

Cothran, D. J., \& Kulinna, P. H. (2007). Students' reports of misbehavior in physical education. Research Quarterly for Exercise and Sport, 78(3), 216-224. http://doi.org/10.5641/193250307X13082490461020

Esteban, R., Fernández Bustos, J. G., Díaz Suárez, A., \& Contreras Jordán, O. R. (2012). Las conductas que alteran la convivencia en las clases de educación física / Behaviours that disturb educational environment in physical education classes. Revista Internacional de Medicina Y Ciencias de La Actividad Física Y Del Deporte, 12(47), 459-472.

Fejgin, N., Talmor, R., \& Erlich, I. (2005). Inclusion and burnout in physical education. European Physical Education Review, 11(1), 29-50. http://doi.org/10.1177/1356336X05049823

Fernandez-Balboa, J. M. (1990). Helping Novice Teachers Handle Discipline Problems. Journal of Physical Education, Recreation \& Dance, 61(7), 5054. http://doi.org/10.1080/07303084.1990.10604580

Finlinson, A. R. (1997). Cooperative Games : Promoting Prosocial Behaviors in Children. Utah State University.

Frydenberg, E. (2004). Coping Competencies: What to Teach and When. Theory into Practice, 43(1), 14-22. https://doi.org/10.1207/s15430421tip4301_3

Gallego, M. A. (2012). Prevención de la disrupción en el aula a través de la gestión democràtica de las normas: tesis doctoral.

Garaigordobil Landazabal, M. (2004). Intervención psicológica en la conducta agresiva y antisocial con niños. Psicothema, 16(3), 429-435.

Gil Espinosa, F. J., Chillón Garzón, P., \& Delgado Noguera, M. A. (2016). Gestión de aula ante Conductas Contrarias a la Convivencia en 
Educación Secundaria Obligatoria Classroom management in problematic behaviors of coexistence in mandatory secondary education. Retos, 30 (c), 2-48. https://doi.org/10.6018/264821

Goyette, R., Doré, R., \& Dion, É. (2000). Pupils' misbehaviors and the reactions and causal attributions of physical education student teachers: $A$ sequential analysis. Journal of Teaching in Physical Education, 20(1), 314. http://doi.org/doi: 10.1123/jtpe.20.1.3

Granero-Gallegos, A., \& Baena-Extremera, A. (2016). Validación española de la versión corta del Physical Education Classroom Instrument para la medición de conductas disruptivas en alumnado de secundaria. Cuadernos de Psicologia Del Deporte, 16(2), 89-98.

Grineski, S. (1996). Cooperative learning in physical education. Champaign, IL: Human Kinetics.

Hastie, P., \& Siedentop, D. (1999). An Ecological Perspective on Physical Education. European Physical Education Review, 5(1), 9-30. http://doi.org/10.1177/1356336X990051002

Kulinna, P. H., Cothran, D. J., \& Recualos, R. (2006). Teachers' reports of student misbehavior in physical education. Reseach Quarterly Exercice Sport, 77(1), 32-40. https://doi.org/10.1080/02701367.2006.10599329

Kulinna, P. H., Cothran, D., \& Recualos, R. (2003). Development of an instrument to measure disruptive behaviour. Measurerement in Physical Education and Exercice Science, 7(1), 25-41. https://doi.org/10.1207/S15327841MPEE0701_3

Latorre, Á. L., \& Romero, J. T. (2014). Protocolo de actuación ante conductas disruptivas. Informacio Psicologica, 0(95), 62-74.

Monzonís, N., \& Capllonch, M. (2014). La educación física en la consecución de la competencia social y ciudadana. Retos. Nuevas Tendencias En Educación Física, Deporte Y Recreación, 2041(25), 180-185.

Navarrete, Lucía \& Ossa, C. (2013). Estilos parentales y calidad de vida familiar en adolescentes con conductas disruptivas. Prensa Médica Latinoamericana - Ciencias Psicológicas 2013, VII (1)(1), 47-56. http://doi.org/ISSN 1688-409-4

Omeñaca, R., \& Ruiz, J. V. (1999). Juegos cooperativos y Educación Física. Barcelona: Paidotribo.

Pérez de Guzmán, V., Amador, L., \& Vargas, M. (2011). Resolución de conflictos en las aulas: un análisis desde la Investigación-Acción. Pedagogía Social. Revista Interuniversitaria, 18(1139-1723), 99-104. https://doi.org/10.7179/PSRI_2011.18.08

Pérez Fuentes, M. del C., Gázquez Linares, J. J., Fernández Baena, R. J. R. J., Molero Jurado, M. del M., Pérez Fuentes del Carmen, M., Gázquez, J. J., ... Molero, M. del M. (2011). Análisis de las publicaciones sobre convivencia escolar en una muestra de revistas de educación en la última década. Aula Abierta, 39(2), 81-90.

Pieron, M. (1999). Para una enseñanza eficaz de las actividades físico deportivas. Barcelona: INDE.

Prat Grau, M., \& Soler Prat, S. (2002). Las posibilidades del juego, la actividad física y el deporte para la mejora de la convivencia. Revista Electrónica Interuniversitaria de Formación Del Profesorado, 81 (28.3)(2), 1-132. http://doi.org/10.1063/1.3080751 
Sánchez, Y. (2012). La educación emocional en permanente del profesorado en la formación permanente del profesorado. I Congreso Virtual Internacional Sobre Innovación Pedagógica Y Praxis Educativa. INNOVAGOGIA, I Congreso, 227-232.

Siedentop, D. (1998). Aprender a enseñar la Educación Física. Barcelona: INDE.

Supaporn, S., Dodds, P., \& Griffin, L. (2003). An Ecological Analysis of Middle School Misbehavior Through Student and Teacher Perspectives. Journal of Teaching in Physical Education, 22, 328-349. https://doi.org/10.1123/jtpe.22.3.328

Uruñuela Nájera, P. M. (2007). Conductas disruptivas en los centros escolares. Revista de Educación de Castilla-La Mancha, 4, 90-93.

Velázquez Callado, C. (2004). Las actividades físicas cooperativas. Una propuesta para la formación en valores a traves de la educación física en las escuelas de educacion básica. (1st ed.). Col. Centro: SECRETARÍA DE EDUCACIÓN PÚBLICA.

White, a G., \& Bailey, J. S. (1990). Reducing disruptive behaviors of elementary physical education students with sit and watch. Journal of Applied Behavior Analysis, 23(3), 353-359. https://doi.org/10.1901/jaba.1990.23353

Número de citas totales / Total references: 34 (100\%)

Número de citas propias de la revista / Journal's own references: 1 (3\%)

Rev.int.med.cienc.act.fís.deporte - vol. 19 - número 76 - ISSN: 1577-0354 


\section{ANEXO I. Evaluación del alumnado en las Unidad Didáctica "Retos cooperativos"}

\begin{tabular}{|c|c|c|c|}
\hline & \multicolumn{1}{|c|}{ Descripción / herramienta } & Tipo & Valor \\
\hline $\begin{array}{c}\text { Evaluación del } \\
\text { RESULTADO }\end{array}$ & $\begin{array}{l}\text { Número de retos superados. Sistema de puntos y niveles. } \\
\text { (ver tabla debajo) }\end{array}$ & Grupal & $30 \%$ \\
\hline $\begin{array}{c}\text { Evaluación del } \\
\text { PROCESO }\end{array}$ & $\begin{array}{l}\text { Participación y actitud en cada uno de los retos y respeto a } \\
\text { las normas. Por medio de una rúbrica. (adjunta más abajo) }\end{array}$ & Grupal & $30 \%$ \\
\hline $\begin{array}{c}\text { AUTOEVALUACIÓN } \\
\text { Cada uno reflexiona sobre su desempeño en la unidad y se } \\
\text { discute al final de la sesión. }\end{array}$ & $\begin{array}{c}\text { Individual- } \\
\text { grupal }\end{array}$ & $20 \%$ \\
\hline $\begin{array}{c}\text { CREAMOS UN } \\
\text { RETOS }\end{array}$ & $\begin{array}{l}\text { Crear un reto cooperativo en grupos de 5 personas y } \\
\text { ponerlo en práctica. Ficha. }\end{array}$ & Grupal & $20 \%$ \\
\hline
\end{tabular}

\begin{tabular}{|c|c|c|c|}
\hline \multicolumn{4}{|c|}{ SISTEMA DE PUNTOS para EVALUAR EL RESULTADO } \\
\hline RETO & ORGANIZACIÓN & RECOMPENSA & NIVEL \\
\hline $\begin{array}{l}\text { 1. Atravesar el aro } \\
\text { horizontal }\end{array}$ & $\begin{array}{l}\text { Gran grupo / intra- } \\
\text { clase }\end{array}$ & 100 puntos & 100 puntos \\
\hline 2. El balón imposible & $\begin{array}{l}\text { Gran grupo / intra- } \\
\text { clase }\end{array}$ & 100 puntos & PRINCIPIANTE \\
\hline $\begin{array}{l}\text { 3. La pelota se va de } \\
\text { marcha }\end{array}$ & $\begin{array}{l}\text { Gran grupo / intra- } \\
\text { clase }\end{array}$ & 100 puntos & \multirow{2}{*}{$\begin{array}{l}300 \text { puntos } \\
\text { INTERMEDIO }\end{array}$} \\
\hline 4. Giro de colchoneta & $\begin{array}{l}\text { Gran grupo / intra- } \\
\text { clase }\end{array}$ & 100 puntos & \\
\hline 5. Paz & $\begin{array}{l}\text { Gran grupo / intra- } \\
\text { clase }\end{array}$ & 100 puntos & \multirow{2}{*}{$\begin{array}{l}500 \text { puntos } \\
\text { AVANZADO }\end{array}$} \\
\hline $\begin{array}{l}\text { 6. Sacar el jamón del } \\
\text { bocadillo }\end{array}$ & $\begin{array}{c}\text { Gran grupo / intra- } \\
\text { clase }\end{array}$ & 100 puntos & \\
\hline 7. El ciempiés viajero & $\begin{array}{l}\text { Gran grupo / intra- } \\
\text { clase }\end{array}$ & 100 puntos & $\begin{array}{l}700 \text { puntos } \\
\text { EXPERTO }\end{array}$ \\
\hline
\end{tabular}


RÚBRICA para evaluar el PROCESO

\begin{tabular}{|c|c|c|c|c|}
\hline $\begin{array}{l}\text { Criterios a tener } \\
\text { en cuenta: }\end{array}$ & Experto & Avanzado & Aprendiz & Inadecuado \\
\hline $\begin{array}{l}\text { El grupo afronta } \\
\text { los retos de forma } \\
\text { autónoma y } \\
\text { demuestran ser } \\
\text { eficaces y } \\
\text { responsables. }\end{array}$ & $\begin{array}{c}\text { Afrontan la tarea de } \\
\text { forma TOTALMENTE } \\
\text { independientes. } \\
\text { ¡Gran organización! } \\
\text { No hay pérdida de } \\
\text { tiempo. }\end{array}$ & $\begin{array}{c}\text { Afrontan la tarea } \\
\text { con BASTANTE } \\
\text { independencia. } \\
\text { ¡Buena } \\
\text { organización! } \\
\text { Pocas pérdidas } \\
\text { de tiempo. }\end{array}$ & $\begin{array}{c}\text { Afrontan la tarea } \\
\text { con SUFICIENCIA. } \\
\text { ¡Tienen problemas } \\
\text { para organizarse! } \\
\text { pero lo logran. Hay } \\
\text { pérdidas de tiempo. }\end{array}$ & $\begin{array}{l}\text { NO son capaces de } \\
\text { organizarse sin la } \\
\text { ayuda del profesor. } \\
\text { ¡Pasan del reto! } \\
\text { Hay bastantes } \\
\text { pérdidas de tiempo. }\end{array}$ \\
\hline $\begin{array}{l}\text { Los miembros del } \\
\text { grupo se apoyan } \\
\text { los unos a los } \\
\text { otros y } \\
\text { demuestran } \\
\text { empatía }\end{array}$ & $\begin{array}{l}\text { TODOS los miembros } \\
\text { se ayudan entre sí. } \\
\text { ¡GRAN ENERGÍA! } \\
\text { Son capaces de } \\
\text { reconocer y } \\
\text { adaptarse a las } \\
\text { dificultades de los } \\
\text { demás. }\end{array}$ & $\begin{array}{l}\text { La MAYORÍA de } \\
\text { miembros se } \\
\text { ayudan entre sí. } \\
\text { ¡HAY ENERGÍA!! } \\
\text { Son capaces de } \\
\text { reconocer y } \\
\text { adaptarse a las } \\
\text { dificultades de los } \\
\text { demás. }\end{array}$ & $\begin{array}{c}\text { ALGUNOS } \\
\text { miembros se } \\
\text { ayudan entre sí. } \\
\text { ¡POCA ENERGÍA!! } \\
\text { Son capaces de } \\
\text { reconocer y } \\
\text { adaptarse con } \\
\text { ayuda del profesor } \\
\text { a las dificultades de } \\
\text { los demás. }\end{array}$ & $\begin{array}{l}\text { POCOS miembros } \\
\text { ayudan a los } \\
\text { demás. } \\
\text { No son capaces de } \\
\text { reconocer y } \\
\text { adaptarse a las } \\
\text { dificultades de los } \\
\text { demás. } \\
\text { Se buscan } \\
\text { culpables o } \\
\text { problemas. }\end{array}$ \\
\hline $\begin{array}{l}\text { El grupo utiliza el } \\
\text { diálogo para } \\
\text { resolver los } \\
\text { desafíos. }\end{array}$ & $\begin{array}{l}\text { SIEMPRE llegan a un } \\
\text { acuerdo y/o } \\
\text { participa TODA la } \\
\text { clase. }\end{array}$ & $\begin{array}{l}\text { CASI SIEMPRE } \\
\text { Ilegan a un } \\
\text { acuerdo y/o } \\
\text { participa gran } \\
\text { parte de la clase. }\end{array}$ & $\begin{array}{c}\text { A VECES llegan a } \\
\text { un acuerdo y/o } \\
\text { participan siempre } \\
\text { los mismos (unos } \\
\text { pocos). }\end{array}$ & $\begin{array}{l}\text { RARA VEZ llegan a } \\
\text { un acuerdo. } \\
\text { Discuten y se } \\
\text { enfadan entre ellos. }\end{array}$ \\
\hline $\begin{array}{l}\text { El grupo resuelve } \\
\text { sus conflictos con } \\
\text { deportividad. }\end{array}$ & $\begin{array}{c}\text { SIEMPRE } \\
\text { ¡Son ejemplares! }\end{array}$ & $\begin{array}{l}\text { La MAYORÍA de } \\
\text { veces. }\end{array}$ & $\begin{array}{l}\text { A VECES no saben } \\
\text { perder. Les cuesta } \\
\text { aceptar la derrota. }\end{array}$ & $\begin{array}{l}\text { RARA VEZ. } \\
\text { Protesta y/o intenta } \\
\text { hacer trampas. }\end{array}$ \\
\hline
\end{tabular}

\section{CREAMOS UN RETO}

\section{№ personas en grupo:}

Material:

\section{Descripción del reto:}

Normas:

"Recuerda comprobar si tu reto es cooperativo a través de las 3 reglas de oro"

Rev.int.med.cienc.act.fís.deporte - vol. 19 - número 76 - ISSN: 1577-0354 